\title{
Effects of quercetin on the expression of MCP-1, MMP-9 and VEGF in rats with diabetic retinopathy
}

\author{
BIN CHEN, TAO HE, YIQIAO XING and TING CAO \\ Department of Ophthalmology, Renmin Hospital of Wuhan University, Wuhan, Hubei 430060, P.R. China
}

Received June 2, 2017; Accepted September 27, 2017

DOI: $10.3892 /$ etm.2017.5275

\begin{abstract}
Diabetic retinopathy, a severe complication of diabetes, is the leading cause of blindness in the developed world. This study investigated the effects of quercetin on levels of monocyte chemoattractant protein-1 (MCP-1), matrix metalloproteinase-9 (MMP-9) and vascular endothelial growth factor (VEGF) in serum of rats with diabetic retinopathy, and explored the functional mechanisms of quercetin in the treatment of diabetic retinopathy. Twenty rats with induced diabetes were divided into a model group and a quercetin group, with 10 rats in each group. Ten healthy rats were also included to serve as a control group. Rats in the quercetin group were treated with an intragastric injection of quercetin $(150 \mathrm{mg} / \mathrm{kg})$, while the same amount of sodium carboxymethyl cellulose (CMCNa) was used for rats in the model group and the control group. The treatment was performed once per day and blood glucose was measured in each group at 0, 10 and 20 weeks after the first treatment. Blood glucose tests showed that quercetin did not reduce blood glucose in rats with diabetes. However, pathological examination showed that quercetin could relieve pathological changes caused by diabetes, such as retinal edema and vacuoles. ELISA results showed that, compared with the control group, levels of MCP-1, MMP-9 and VEGF in the model group were significantly increased $(\mathrm{P}<0.01)$. No significant difference in serum MCP-1 content was found between the model group and the quercetin group, but levels of MMP-9 and VEGF were significantly decreased in the quercetin group $(\mathrm{P}<0.01)$. Results of RT-PCR and western blot analysis showed that, compared with the control group, levels of MCP-1, MMP-9 and VEGF mRNA and protein in the retinal tissue of rats in the model group were significantly increased $(\mathrm{P}<0.01)$. No significant differences in expression levels of MCP-1 mRNA and protein were found between the model group and the quercetin group, but levels of MMP-9 and
\end{abstract}

Correspondence to: Dr Yiqiao Xing, Department of Ophthalmology, Renmin Hospital of Wuhan University, 238 Jiefang Road, Wuhan, Hubei 430060, P.R. China

E-mail: xs44cz@163.com

Key words: diabetic retinopathy, quercetin, monocyte chemoattractant protein-1, matrix metalloproteinase-9, vascular endothelial growth factor
VEGF mRNA and protein were significantly decreased in the quercetin group $(\mathrm{P}<0.01)$. Quercetin has a certain therapeutic effect on rats with diabetic retinopathy and its effect may be achieved by reducing the expression of MMP-9 and VEGF, but not the inflammatory mediator, MCP-1.

\section{Introduction}

The incidence of diabetes is alarmingly high and has shown no signs of decreasing its upward trend. Beyond the burden of disease that diabetes itself presents, complications of diabetes can also seriously affect a patient's health. Among complications of diabetes, diabetic retinopathy is the most serious eye disease as it may cause blindness. The primary cause of diabetic retinopathy is long-term hyperglycemia; that is, diabetic retinopathy is correlated with the severity and duration of hyperglycemia (1). It is predicted that the number of patients with diabetes in the United States will reach 25 million by 2030 , and the number of patients with diabetic retinopathy by then will reach 300 million worldwide (2).

Development of diabetic retinopathy is related to a variety of factors, including neovascularization, in which vascular endothelial growth factor (VEGF) plays a pivotal role in every step $(3,4)$. VEGF can promote the migration, division and proliferation of endothelial cells; improve the activity of plasma plasminogen activators; and can activate gene expression in endothelial cells, leading to neovascularization $(5,6)$. Studies have shown that matrix metalloproteinase-9 (MMP-9) also plays an important role in neovascularization. In addition, monocyte chemoattractant protein-1 (MCP-1) plays essential roles in the progression of various complications of diabetes (7-9).

Based on the pathogenesis of diabetic retinopathy, a variety of drugs have been developed, such as antioxidants, aldose reductase inhibitors, antiplatelet drugs, and drugs that can inhibit the formation of advanced glycation end products; however, the application of these drugs are still challenged by the medical community due to uncertain efficacy and adverse side effects (10). The treatment of diabetes has a long history in Chinese medicine. Studies have shown that Chinese medicine can significantly improve diabetes and its related complications. Pharmacological studies on traditional Chinese medicine also provide a reference for further use of traditional Chinese medicine in treatment of diabetes (11). Quercetin, as a component of traditional Chinese medicine, functions as an antioxidant and in aldose reductase inhibition. In this study, 
the effects of quercetin on rats with diabetic retinopathy were investigated by assessing its influence on various molecular factors implicated in the pathogenesis of the disease.

\section{Materials and methods}

Materials and reagents. Quercetin and streptozotocin (STZ) were purchased from Sigma (New York, NY, USA); MCP-1, MMP-9, VEGF and an enzyme-linked immunosorbent assay (ELISA) kit were purchased from Nanjing Jiancheng Bioengineering Institute (Nanjing, China); rabbit anti-rat primary polyclonal antibodies of MCP-1, MMP-9 and VEGF (dilution, 1:500; cat. nos. 10194-1-AP, 14552-1-AP and 190031-AP) and Goat anti-rabbit HRP-labeled secondary polyclonal antibody were purchased from Proteintech (Wuhan, China) (dilution, 1:2,000; cat. no. SA00001-2); primer synthesis, reverse transcription kit and a real-time PCR kit were purchased from Takara (Dalian, China); and a BCA protein quantitation kit and cell lysate was purchased from Beyotime (Nantong, China).

Experimental animal grouping and sample collection. Thirty healthy male Sprague-Dawley rats (180-220 g) were used in the study (SLAC Laboratory Animal Co., Ltd., Shanghai, China). Twenty of the rats were randomly selected and fasted for $24 \mathrm{~h}$, followed by intraperitoneal injection of STZ dissolved in a $2 \%$ citric acid buffer (60 mg/kg dosage). Blood was extracted from the tail vein $72 \mathrm{~h}$ later and all 20 rats showed a blood glucose level $>16.7 \mathrm{mmol} / 1$, indicating successfully established diabetes. The other 10 rats were treated with an intraperitoneal injection of the same amount of $2 \%$ citric acid buffer to serve as a control. The 20 rats with diabetes were further divided randomly into 2 groups, including a model group and a quercetin group, with 10 rats in each group. Rats in the quercetin group were treated with an intragastric injection of quercetin $(150 \mathrm{mg} / \mathrm{kg})$, while the same amount of sodium carboxymethyl cellulose (CMCNa) was used for rats in the model group and the control group. Treatment was performed once per day. Blood samples were extracted from the tail vein of rats in each group at 0,10 and 20 weeks after the first treatment and a blood glucose meter was used to measure blood glucose. After treatment for 20 weeks, the rats were anesthetized with $10 \%$ chloral hydrate $(3 \mathrm{mg} / \mathrm{kg})$. Blood was extracted from the inferior cava vein and the rats were then sacrificed. Eyeballs were collected and the ocular anterior segment and retina were removed and stored at $-80^{\circ} \mathrm{C}$ to be used later for RT-PCR and western blot analysis. The right eyeball was fixed in $4 \%$ paraformaldehyde solution, followed by dehydration by passing over a series of graded ethanol concentrations. Dehydrated eyeballs were then treated with xylene to make them transparent. After paraffin embedding, the eyeballs were cut into sections for $\mathrm{H} \& \mathrm{E}$ staining. The study was approved by the Ethics Committee of Renmin Hospital of Wuhan University (Hubei, China).

ELISA to detect levels of MCP-1, MMP-9 and VEGF in serum of rats. The levels of MCP-1, MMP-9 and VEGF in the serum of each group were detected by ELISA. All operations were performed in strict accordance with the instructions of the kit. Each sample was tested three times with two technique repetitions performed each time.
Table I. Primers for MCP-1, MMP-9 and VEGF used in RT-PCR.

\begin{tabular}{ll} 
Genes & \multicolumn{2}{c}{ Primer sequences } \\
\hline VEGF & F: 5'-TTTCGGGAACTAGACCTCTCACC-3' \\
& R: 5'-CTTCATGTCAGGCTTTCTGGATT-3' \\
MMP-9 & F: 5'-CCCCCGAGACCTGAAAACCT-3' \\
& R: 5'-TGATGTTATGATGGTGCCACTTGA-3' \\
MCP-1 & F: 5'-CCCACTCACCTGCTGCTACTC-3' \\
& R: 5'-AGAAGTGCTTGAGGTGGTTGTG-3' \\
GAPDH & F: 5'-GCACCGTCAAGGCTGAGAAC-3' \\
& R: 5'-TGGTGAAGACGCCAGTGGA-3'
\end{tabular}

MCP-1, monocyte chemoattractant protein-1; MMP-9, matrix metalloproteinase-9; VEGF, vascular endothelial growth factor.

RT-PCR to detect expression of MCP-1, MMP-9 and VEGF $m R N A$ in rat retina. Retinal tissue of the rats was collected and total RNA was extracted. Concentration and purity of RNA were measured and only the samples with a ratio of A260/A280 between 1.8 and 2.0 were used. Reverse transcription was performed according to the instructions of the kit and the synthesized cDNA was used as a template for RT-PCR reaction. All primer sequences are listed in Table I. PCR reaction conditions were as follows: $94^{\circ} \mathrm{C}$ for $5 \mathrm{~min}$, followed by 30 cycles of $94^{\circ} \mathrm{C}$ for $30 \mathrm{sec}$, then $57^{\circ} \mathrm{C}$ for $30 \mathrm{sec}, 72^{\circ} \mathrm{C}$ for $30 \mathrm{sec}$ and $72^{\circ} \mathrm{C}$ for $5 \mathrm{~min}$. $\mathrm{Ct}$ values were processed using the $2^{-\Delta \mathrm{Ct}}$ method: $\Delta \mathrm{Ct}$ $($ target gene $)=\mathrm{Ct}($ target gene $)-\mathrm{Ct}($ control gene $)$.

Western blot analysis to detect the expression of MCP-1, $M M P-9$ and VEGF in rat retina. Frozen rat retinal tissue was used to extract total protein content. Protein concentration was then measured. After processing, protein samples $(50 \mu \mathrm{g})$ were subjected to SDS-PAGE electrophoresis, followed by transfer to a PVDF membrane. The membrane was blocked with blocking solution at room temperature for $1 \mathrm{~h}$, followed by incubation with the primary antibody overnight at $4^{\circ} \mathrm{C}$. After washing with TTBS, the membrane was incubated with the secondary antibody $(1: 2,000)$ at room temperature for $1 \mathrm{~h}$. After washing with TTBS, color development was performed and images were taken.

Statistical analysis. The data are expressed as mean \pm standard deviation and processed using SPSS 17.0 (IBM Corp., New York, NY, USA). Comparisons between 2 groups were performed using t-test and $\mathrm{P}<0.05$ was considered to be statistically significant.

\section{Results}

Effects of quercetin on blood glucose in rats with diabetes. The changes in blood glucose in each group are shown in Table II. Compared with the control group, blood glucose was significantly increased in the model group and the quercetin group $(\mathrm{P}<0.01)$. During the whole experiment, no significant difference in blood glucose levels was found between the model 

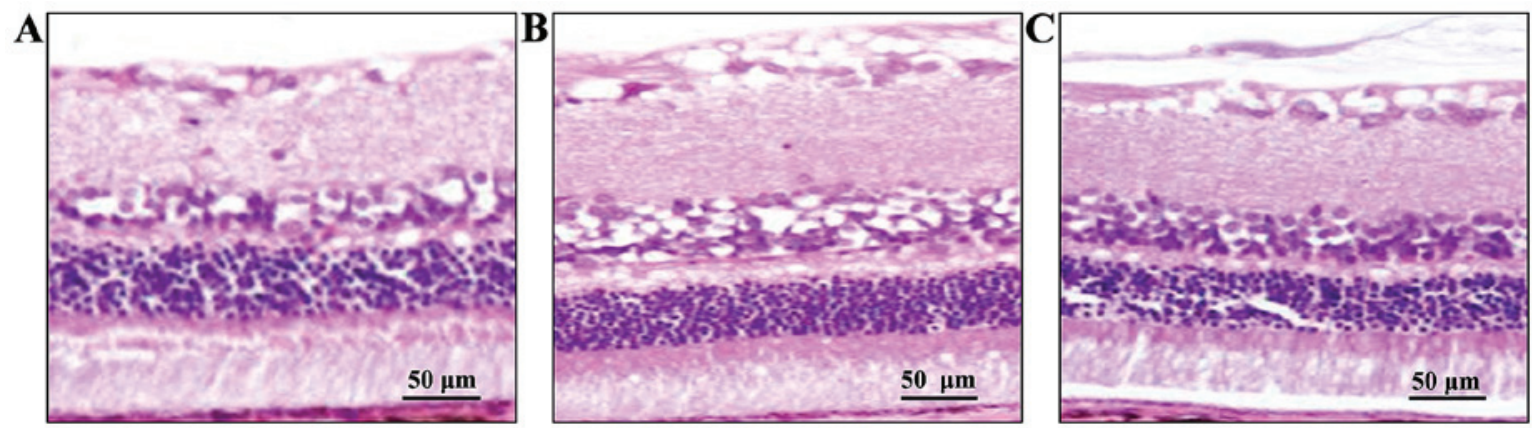

Figure 1. Histological changes of retina in each group. (A) Control group: retinal tissue was intact. (B) Diabetes group: retinal tissue in the diabetes group was thinner than that of the control group, structure was looser and cell vacuolization was observed. (C) Quercetin group: mild edema in retinal tissue and cell vacuolization was observed.
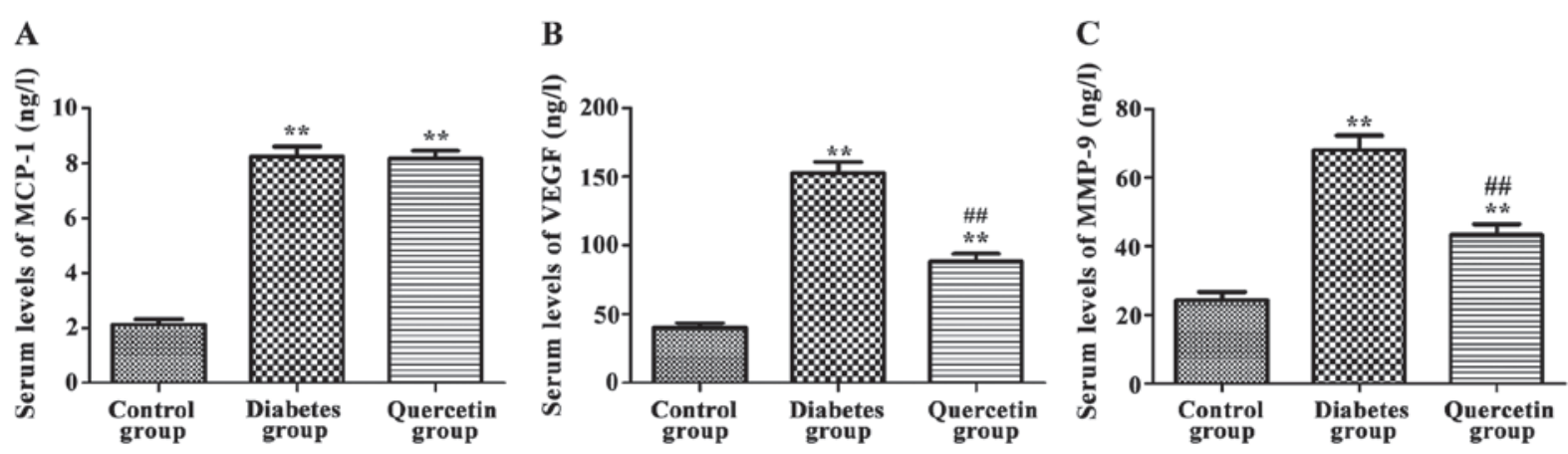

Figure 2. Effects of quercetin on serum levels of monocyte chemoattractant protein-1 (MCP-1), matrix metalloproteinase-9 (MMP-9) and vascular endothelial growth factor (VEGF). (A) Serum MCP-1 levels in rats of each group. (B) Serum MMP-9 levels in rats of each group and (C) serum VEGF levels in rats of each group. Compared with the control group, serum levels of MCP-1, MMP-9 and VEGF were significantly increased in the diabetes group. Compared with the diabetes group, serum levels of MMP-9 and VEGF were significantly decreased in the quercetin group, but no significant changes in serum level of MCP-1 were observed. ${ }^{* *} \mathrm{P}<0.01$ compared with control group; ${ }^{\# \#} \mathrm{P}<0.01$ compared with diabetes group.

Table II. Changes in blood glucose levels (mmol/l) in each group.

\begin{tabular}{cccc}
\hline $\begin{array}{l}\text { Time } \\
\text { (weeks) }\end{array}$ & $\begin{array}{c}\text { Control } \\
\text { group }\end{array}$ & $\begin{array}{c}\text { Model } \\
\text { group }\end{array}$ & $\begin{array}{c}\text { Quercetin } \\
\text { group }\end{array}$ \\
\hline 0 & $4.68 \pm 0.21$ & $4.75 \pm 0.16$ & $4.81 \pm 0.12$ \\
10 & $4.71 \pm 0.14$ & $23.33 \pm 3.15^{\mathrm{a}}$ & $22.92 \pm 3.9^{\mathrm{a}}$ \\
20 & $4.82 \pm 0.19^{\mathrm{a}}$ & $24.22 \pm 2.9^{\mathrm{a}}$ & $24.17 \pm 3.4^{\mathrm{a}}$ \\
\hline
\end{tabular}

${ }^{\mathrm{a}} \mathrm{P}<0.01$ compared with control group.

group and the quercetin group $(\mathrm{P}>0.05)$, indicating that quercetin has no effects on blood glucose levels.

Effects of quercetin on histological changes of rat retina. Retinal tissue was observed under optical microscope (Olympus, Tokyo, Japan). As shown in Fig. 1 retinal tissue in the control group was intact and the layers of retina were clear and regularly arranged. Retinal tissue in the model group was thinner than that of the control group and the structure was loose and cell vacuolization was observed. Compared with the model group, histological changes of the retina were improved in the quercetin group, while edema and cell vacuolization were still observed compared with the control group.
Effects of quercetin on serum levels of MCP-1, MMP-9 and $V E G F$. As shown in Fig. 2, compared with control group, serum levels of MCP-1, MMP-9 and VEGF were significantly increased in the model group $(\mathrm{P}<0.01)$. Compared with the model group, serum levels of MMP-9 and VEGF were significantly decreased in the quercetin group $(\mathrm{P}<0.01)$, but no significant changes in serum levels of MCP-1 were observed $(\mathrm{P}>0.05)$, indicating that quercetin could significantly decrease serum levels of MMP-9 and VEGF, but not MCP-1.

Effects of quercetin on expression of MCP-1, MMP-9 and VEGF $m R N A$ in rat retina. As shown in Fig. 3, compared with the control group, expression of MCP-1, MMP-9 and VEGF mRNA of the retina were significantly increased in the model group $(\mathrm{P}<0.01)$. Compared with the model group, the expression levels of MMP-9 and VEGF mRNA were significantly decreased in the quercetin group $(\mathrm{P}<0.01)$, but no significant changes in the expression level of MCP-1 was observed $(\mathrm{P}>0.05)$, indicating that quercetin could significantly decrease the expression of MMP-9 and VEGF mRNA, but not MCP-1 mRNA.

Effects of quercetin on the expression of MCP-1, MMP-9 and $V E G F$ protein in rat retina. As shown in Fig. 4, compared with control group, the expression of MCP-1, MMP-9 and VEGF protein in retina were significantly increased in the model group $(\mathrm{P}<0.01)$. Compared with the model group, expression levels 

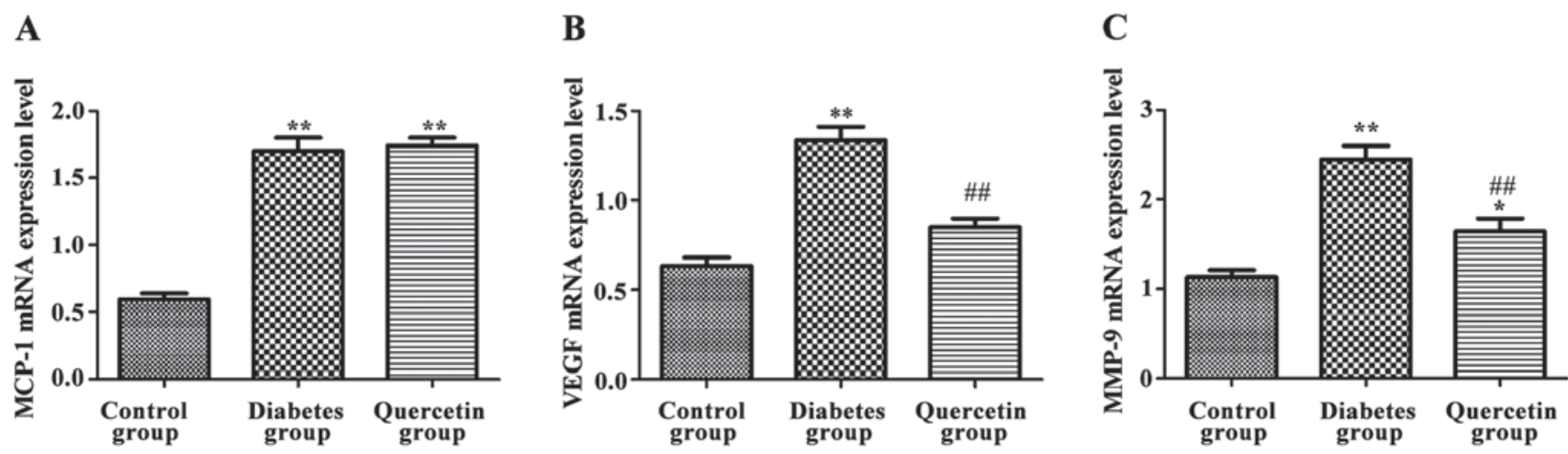

Figure 3. Effects of quercetin on the expression of monocyte chemoattractant protein-1 (MCP-1), matrix metalloproteinase-9 (MMP-9) and vascular endothelial growth factor (VEGF) mRNA in rat retina. (A) Expression levels of MCP-1 mRNA in rat retina. (B) Expression levels of MMP-9 mRNA in rat retina. (C) Expression levels of VEGF mRNA in rat retina. Compared with the control group, expression levels of MCP-1, MMP-9 and VEGF mRNA in the retina were significantly increased in the diabetes group. Compared with the diabetes group, expression levels of MMP-9 and VEGF mRNA were significantly decreased in the quercetin group, but no significant changes in expression levels of MCP-1 were observed. ${ }^{* *} \mathrm{P}<0.01$ compared with the control group; ${ }^{\# \prime} \mathrm{P}<0.01$ compared with the diabetes group.

$\mathbf{A}$

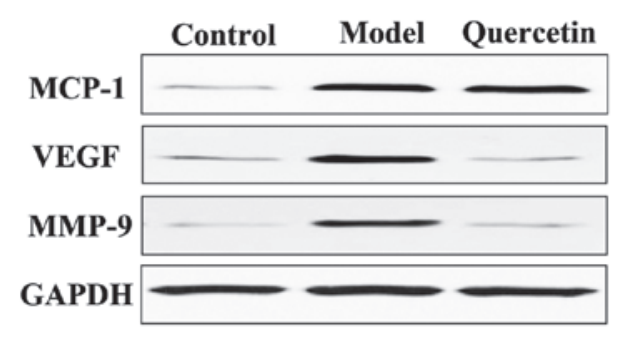

B

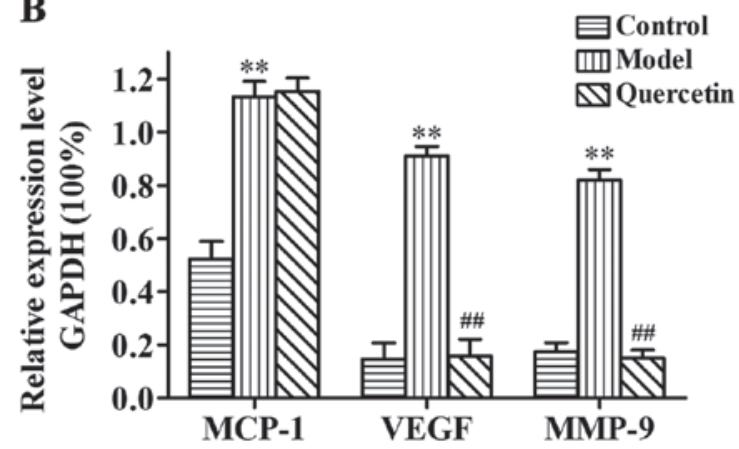

Figure 4. Expression of monocyte chemoattractant protein-1 (MCP-1), matrix metalloproteinase-9 (MMP-9) and vascular endothelial growth factor (VEGF) protein in rat retina as detected by western blot analysis. (A) Representative result of western blot analysis, with relative expression levels of MCP-1, MMP-9 and VEGF protein in each group. (B) Compared with the control group, expression levels of MCP-1, MMP-9 and VEGF protein in the retina were significantly increased in the diabetes group. Compared with the diabetes group, expression levels of MMP-9 and VEGF protein were significantly decreased in the quercetin group, but no significant changes in the expression levels of MCP-1 were observed. ${ }^{* *} \mathrm{P}<0.01$ compared with the control group; ${ }^{\# \# ~} \mathrm{P}<0.01$ compared with the diabetes group.

of MMP-9 and VEGF protein were significantly decreased in the quercetin group $(\mathrm{P}<0.01)$, but no significant changes in the expression levels of MCP-1 were observed ( $\mathrm{P}>0.05)$, indicating that quercetin could significantly decrease the expression levels of MMP-9 and VEGF protein, but not the inflammatory mediator protein, MCP-1.

\section{Discussion}

Diabetic retinopathy is one of the most common and severe complications of diabetes. It has been shown that diabetic retinopathy is the main cause of blindness in developed countries. The disease is characterized by early high perfusion that can cause significant expansion and deformation of capillaries, leading to damage to the retina barrier. Progression of the disease is characterized by capillary occlusion, bleeding and even the formation of new blood vessels (12). Extensive research into the pathogenesis, clinical treatment and other aspects of diabetic retinopathy has been conducted by researchers. However, the pathogenesis of diabetic retinopathy caused by early diabetes is still unclear and effective clinical treatment is still lacking (13).
In the course of diabetes progression, disorders of glycometabolism are the leading causes of diabetic retinopathy. Other molecular factors, such as MMPs, inflammatory mediators (e.g., MCP-1) and VEGF are also involved (14-16). The main process of neovascularization includes the degradation of extracellular matrix by vascular endothelial cells, migration and proliferation of endothelial cells and the synthesis of new matrix components (17). Formation of new blood vessels, which plays a pivotal role in the progression of diabetic retinopathy, can also fundamentally affect the development of the body, wound healing and reproduction (18). In addition, degradation of the basement membrane is also an important step in the formation of new blood vessels. MMP plays an important role in the reconstruction of the basement membrane. According to the different structures, MMP can be divided into two categories: membrane-type MMP and secreted-type MMP. There are three types of secreted-type MMP, including matrixase, such as MMP-3 and MMP-10, collagenase, such as MMP-1 and MMP-8, and gelatinase, such as MMP-2 and MMP-9. Studies have shown that MMP plays an important role in the formation of neovascularization, especially MMP-9, which is involved in the formation of vascular endothelial cells during the 
development of new blood vessels $(19,20)$. MCP-1, as a member of the family of inflammatory factors and chemokines, exerts its roles mainly through paracrine and autocrine. MCP-1 can induce the migration and infiltration of macrophages and monocytes and plays an important role in the activation of mononuclear phagocytic cells and inflammation (21). Studies have shown that MCP-1 expression is increased in the vitreous, retinal vessel wall and related cells in diabetic retinopathy. In addition, expression levels of TNF- $\alpha$ are positively correlated with MCP-1 expression (22).

In this study, STZ was used to establish a diabetic rat model and increased blood glucose levels indicate the successful establishment of the model. The aim of this study was to investigate the effects of quercetin on the expression of MCP-1, MMP-9 and VEGF in rats with diabetic retinopathy. Results showed no significant differences in blood glucose levels between the model group and the quercetin group, indicating that quercetin has no effects in reducing blood glucose. Therefore, the effects of quercetin on diabetic retinopathy were not achieved by reducing the level of blood glucose. Histological results from this study found that quercetin can reduce the pathological changes caused by diabetes, such as retinal tissue edema and cell vacuolization. ELISA results showed that, compared with rats in the control group, serum levels of MCP-1, MMP-9 and VEGF were significantly increased in the model group. Compared with the model group, serum levels of MMP-9 and VEGF were significantly decreased in the quercetin group, but no significant differences in serum levels of MCP-1 were found between these two groups. RT-PCR and western blot analysis showed that, compared with the control group, the expression of MCP-1, MMP-9 and VEGF mRNA and protein in the retina were significantly increased in the model group. Compared with the model group, expression levels of MMP-9 and VEGF mRNA and protein were significantly decreased in quercetin group, but no significant differences in the expression of MCP-1 mRNA and protein were found between these two groups.

In conclusion, the findings of this study indicate that quercetin can protect rats from the damage caused by STZ-induced diabetic retinopathy, and its effects may be achieved by downregulating expression levels of MMP-9 and VEGF, but not MCP-1.

\section{References}

1. Funatsu H, Yamashita H, Nakamura S, Mimura T, Eguchi S, Noma $\mathrm{H}$ and Hori S: Vitreous levels of pigment epitheliumderived factor and vascular endothelial growth factor are related to diabetic macular edema. Ophthalmology 113: 294-301, 2006.

2. Qaum T, Xu Q, Joussen AM, Clemens MW, Qin W, Miyamoto K, Hassessian H, Wiegand SJ, Rudge J, Yancopoulos GD, et al: VEGF-initiated blood-retinal barrier breakdown in early diabetes. Invest Ophthalmol Vis Sci 42: 2408-2413, 2001.

3. Kant S, Seth G and Anthony K: Vascular endothelial growth factor-A (VEGF-A) in vitreous fluid of patients with proliferative diabetic retinopathy. Ann Ophthalmol (Skokie) 41: 170-173, 2009.
4. Eichler W, Yafai Y, Keller T, Wiedemann P and Reichenbach A: PEDF derived from glial Müller cells: A possible regulator of retinal angiogenesis. Exp Cell Res 299: 68-78, 2004.

5. Jorge R, Costa RA, Calucci D, Cintra LP and Scott IU: Intravitreal bevacizumab (Avastin) for persistent new vessels in diabetic retinopathy (IBEPE study). Retina 26: 1006-1013, 2006.

6. Zhang SX, Wang JJ, Gao G, Parke K and Ma JX: Pigment epithelium-derived factor downregulates vascular endothelial growth factor (VEGF) expression and inhibits VEGF-VEGF receptor 2 binding in diabetic retinopathy. J Mol Endocrinol 37: $1-12,2006$.

7. Kim JO, Lee GD, Kwon JH and Kim KS: Anti-diabetic effects of new herbal formula in neonatally streptozotocin-induced diabetic rats. Biol Pharm Bull 32: 421-426, 2009.

8. Forsyth PA, Wong H, Laing TD, Rewcastle NB, Morris DG, Muzik H, Leco KJ, Johnston RN, Brasher PM, Sutherland G, et al: Gelatinase-A (MMP-2), gelatinase-B (MMP-9) and membrane type matrix metalloproteinase-1 (MT1-MMP) are involved in different aspects of the pathophysiology of malignant gliomas. Br J Cancer 79: 1828-1835, 1999.

9. Robison WG Jr, Laver NM, Jacot JL, Chandler ML, York BM and Glover JP: Efficacy of treatment after measurable diabeticlike retinopathy in galactose-fed rats. Invest Ophthalmol Vis Sci 38: 1066-1073, 1997

10. Joussen AM, Poulaki V, Mitsiades N, Kirchhof B, Koizumi K, Döhmen S and Adamis AP: Nonsteroidal anti-inflammatory drugs prevent early diabetic retinopathy via TNF-alpha suppression. FASEB J 16: 438-440, 2002.

11. Zhang TT and Jiang JG: Active ingredients of traditional Chinese medicine in the treatment of diabetes and diabetic complications. Expert Opin Investig Drugs 21: 1625-1642, 2012.

12. Aiello LP: Angiogenic pathways in diabetic retinopathy. N Engl J Med 353: 839-841, 2005.

13. Kim JH, Kim JH, Yu YS, Cho CS and Kim KW: Blockade of angiotensin II attenuates VEGF-mediated blood-retinal barrier breakdown in diabetic retinopathy. J Cereb Blood Flow Metab 29: 621-628, 2009.

14. Adamiec-Mroczek J,Oficjalska-Młyńczak J and Misiuk-Hojło M: Proliferative diabetic retinopathy - The influence of diabetes control on the activation of the intraocular molecule system. Diabetes Res Clin Pract 84: 46-50, 2009.

15. Noda K, Ishida S, Inoue M, Obata K, Oguchi Y, Okada Y and Ikeda E: Production and activation of matrix metalloproteinase-2 in proliferative diabetic retinopathy. Invest Ophthalmol Vis Sci 44: 2163-2170, 2003.

16. EI-Asrar AM: Missotten L and Geboes K: Expression of cycloxygenase- 2 and downstream enzymes in diabetic fibrovaseular epiretinal membranes. Br J Ophthalmol 92: 1534-1539, 2008.

17. Watanabe D: Erythropoietin as a retinal angiogenic factor in proliferative diabetic retinopathy. Nippon Ganka Gakkai Zasshi 111: 892-898, 2007 (In Japanese).

18. Siekmann AF and Lawson ND: Notch signalling limits angiogenic cell behaviour in developing zebrafish arteries. Nature 445: 781-784, 2007.

19. Du Y, Sarthy VP and Kern TS: Interaction between NO and COX pathways in retinal cells exposed to elevated glucose and retina of diabetic rats. Am J Physiol Regul Integr Comp Physiol 287: R735-R741, 2004.

20. Kern TS: Contributions of inflammatory processes to the development of the early stages of diabetic retinopathy. Exp Diabetes Res 2007: 95103, 2007.

21. Wakabayashi Y, Usui Y, Okunuki Y, Kezuka T, Takeuchi M, Iwasaki T, Ohno A and Goto $\mathrm{H}$ : Increases of vitreous monocyte chemotactic protein 1 and interleukin 8 levels in patients with concurrent hypertension and diabetic retinopathy. Retina 31: 1951-1957, 2011.

22. Dong N, Li X, Xiao L, Yu W, Wang B and Chu L: Upregulation of retinal neuronal MCP-1 in the rodent model of diabetic retinopathy and its function in vitro. Invest Ophthalmol Vis Sci 53: 7567-7575, 2012. 